\title{
ETAPAS DEL CAMBIO, BENEFICIOS Y BARRERAS EN ACTIVIDAD FÍSICA Y CONSUMO DE FRUTAS Y VERDURAS EN ESTUDIANTES UNIVERSITARIOS DE SANTIAGO DE CHILE
}

\section{STAGES OF CHANGE, BENEFITS AND BARRIERS ON PHYSICAL ACTIVITY AND FRUIT AND VEGETABLES INTAKE IN CHILEAN UNIVERSITY STUDENTS}

\author{
Sonia Olivares C., Lydia Lera M., Nelly Bustos Z. \\ Instituto de Nutrición y Tecnología de los Alimentos (INTA). Universidad de Chile.
}

Este trabajo fue recibido el 28 de Noviembre de 2007 y aceptado para ser publicado el 19 de Marzo de 2008.

Dirigir la correspondencia a:

Profesora Sonia Olivares

Prof. Asociada

INTA, Universidad de Chile

El Líbano 5524 - Macul

Santiago, Chile

Fono: 56-2-9781437

Fax: 56-2-2214030

e-mail: solivare@inta.cl 


\begin{abstract}
As baseline for health promotion and education interventions design, the stages of change, benefits and barriers in physical activity and fruit and vegetables intake were studied in 449 university students from Santiago. Instruments internationally validated and adapted to the country were applied. In physical activity, men were significant more active than women. Benefits like "I feel well" o "it is entertaining" were more common in men and "to loose weight" was most common in women. Lack of time was the main barrier in both genders. 58\% ate 1-2 portions of fruit and vegetables/daily and only 5\% ate 5 portions daily. The main benefit associated with fruit and vegetables intake was "to be healthy". Women were more motivated. "I am lazy for preparing them" or "I forget to eat them" were the most common barriers in both genders. These results will be useful in health promotion and education interventions with university students.
\end{abstract}

Key words: university students, stages of change, attitudes, physical activity, fruit \& vegetables intake.

\title{
INTRODUCCIÓN
}

Según la Encuesta Nacional de Salud (ENS), realizada por el Ministerio de Salud en una muestra representativa de la población chilena el año 2003 (1), en jóvenes de 17 a 24 años, la prevalencia de sobrepeso y obesidad alcanzaba al 25,4\%; la hipertensión al 5,6\% y el colesterol elevado al 6\%; en comparación con el 61\% de sobrepeso y obesidad; 33,7\% de hipertensión y 35,4\% de colesterol elevado en la población general (17 a 65 años). El sedentarismo en tanto, muy elevado en todos los grupos, afectaba al $82 \%$ de los jóvenes y al 90,8\% de la población general (1).

Los estudiantes universitarios constituyen uno de los grupos objetivos de la Política Nacional de Promoción de Salud (2). En este contexto, h Guía para Universidades Saludables (3), señala que "promover cambios en la situación de salud del país a través de las generaciones de estudiantes que egresan de las universidades e institutos de educación superior, es una forma efectiva de fomentar cambios a favor del bienestar y la salud en las instituciones y comunidades en las que trabajarán". 
Promover el aumento de la actividad física y la alimentación saludable en todos los grupos de edad constituyen los ejes centrales de las recomendaciones de la OMS, la FAO y el Fondo Internacional para la Investigación del Cáncer (WCRF), entre otras, quienes difunden la evidencia científica y entregan las bases para el desarrollo de iniciativas en estos temas a nivel mundial (4-6).

La falta de actividad física ha sido reconocida como un factor de riesgo independiente en la aparición de enfermedades no transmisibles, lo que ha motivado la creación de estrategias internacionales para motivar su aumento en todos los grupos de la población $(4,7)$.

Las recomendaciones para promover y mantener la salud en los adultos sanos, indican que éstos necesitan realizar un mínimo de 30 minutos de actividad física moderada e intensa, cinco días a la semana, combinando ejercicios de distinta intensidad, por ejemplo, 20 minutos de actividad moderada y 10 minutos de actividad intensa, para aumentar la respiración y el ritmo cardíaco de manera sustancial (8). La Guía para una Vida Saludable publicada por el Ministerio de Salud, el INTA y Vida Chile en el año 2007 (9), recomienda a la población chilena "caminar al menos 30 minutos diarios" y "realizar ejercicios 30 minutos 3 veces por semana", entre otros mensajes.

El bajo consumo de frutas y verduras ha sido reconocido como factor de riesgo de las enfermedades cardiovasculares y algunos tipos de cáncer (4-6, 10). Las Guías Alimentarias para la población chilena (9), incluyen el mensaje "Come al menos 2 platos de verduras y 3 frutas de distintos colores cada día" en concordancia con las recomendaciones internacionales de consumir al menos 5 porciones diarias de estos alimentos $(4,6,11)$. Aunque Chile es un importante productor y exportador de frutas y hortalizas (12), el consumo de la población en prácticamente todos los grupos de edad alcanza aproximadamente a la mitad de lo indicado por la OMS, la que recomienda consumir al menos 400 gramos diarios (4).

Las causas que motivan este menor consumo han sido asociadas al cambio en el estilo de alimentación de los chilenos en los últimos 20 años, con un aumento significativo en el consumo de alimentos procesados de alta densidad energética, ricos en grasas totales, grasas 
saturadas, azúcar, sal y un bajo contenido de fibra dietética, reconocidos entre los principales factores de riesgo, junto al sedentarismo, de la creciente prevalencia de sobrepeso y obesidad observada en el país en los últimos años (13). El elevado consumo de este tipo de productos ha sido atribuido a exitosas estrategias de publicidad dirigidas a los niños y jóvenes, que los han colocado al alcance de toda la población, con una gran oferta, bajos precios y alto prestigio social $(14,15)$.

Actualmente, con la creación de la "Corporación 5 al Día Chile" en el año 2006 (16), se han iniciado campañas que promueven el consumo de frutas y verduras a través de los medios masivos de comunicación. Estas campañas han sido realizadas por la propia Corporación y por algunos de sus miembros asociados, en especial, una de hs principales cadenas nacionales de supermercados. Estudios internacionales han mostrado que los niños y los grupos de menor nivel socioeconómico son muy sensibles a la publicidad de los alimentos a través de los medios masivos, en especial la televisión, los que afectan su elección, compra y consumo, por lo que las campañas orientadas a lograr que la población tome conciencia de la importancia de consumir más frutas y verduras adquieren especial relevancia $(14,15)$.

El estudio de las etapas del cambio (17), que indican donde se encuentran las personas en relación a una conducta en particular, así como de las motivaciones que podrían orientar las campañas de promoción, educación o publicidad social, y la identificación de las barreras o costos que dificultan su adopción en los grupos objetivo, han sido escasamente estudiadas en el país. En actividad física y consumo de frutas y verduras, en esencial realizar estudios cualitativos y cuantitativos sobre las percepciones, creencias y valores actuales de los distintos grupos objetivo, con el fin de segmentar audiencias y diseñar productos, mensajes y estrategias ajustados a las características que comparten(18-21).

En Chile, las iniciativas destinadas a reducir la prevalencia de obesidad y aumentar la actividad física en la población se han abordado principalmente desde la perspectiva de la promoción de la salud, básicamente realizada por los Centros de Atención Primaria y algunas escuelas, en acciones aisladas y esporádicas, las que hasta el momento no han logrado avances en la dirección de las metas sanitarias fijadas por el gobierno para el año 2010, de reducir el 
sedentarismo en toda la población y la prevalencia de sobrepeso y obesidad en preescolares, escolares y embarazadas (2).

El objetivo de este trabajo es determinar las etapas del cambio y la percepción sobre los beneficios y barreras de realizar al menos 30 minutos de actividad física 5 días a la semana y consumir 5 porciones diarias de frutas y verduras en estudiantes universitarios chilenos, como parte de una línea de trabajo que inclu ye a profesores de enseñanza básica, profesionales de salud, padres de escolares y escolares de distinto nivel socioeconómico (NSE).

\section{SUJETOS Y MÉTODO}

Se trata de un estudio descriptivo de corte transversal, en el que se aplicó una encuesta sobre etapas del cambio, percepción de beneficios y barreras en actividad física y consumo de frutas y verduras a 449 estudiantes de la Universidad de Chile, en el año 2007. El tamaño de la muestra (355 individuos) se calculó sobre la base de la prevalencia de sedentarismo en los jóvenes chilenos (82\%) (1), con un nivel de significación del 5\% y una precisión del 4\%. Las características de la muestra se presentan en la tabla 1.

\section{TABLA 1}

\section{Distribución de los estudiantes universitarios encuestados}

$\begin{array}{lc}\text { Hombres (número y porcentaje) } & 188(41,9 \%) \\ \text { Mujeres (número y porcentaje) } & 261(59,1 \%) \\ \text { Total (número y porcentaje) } & 449(100 \%) \\ \text { Rango de edad } & 19-24 \text { años } \\ \text { Estatura promedio } \pm \text { DE hombres (en cm) } & 175,2 \pm 7,1 \\ \text { Estatura promedio } \pm \text { DE mujeres (en cm) } & 161,9 \pm 5,6 \\ \text { Peso promedio } \pm \text { DE hombres (en kg) } & 74,1 \pm 10 \\ \text { Peso promedio } \pm \text { DE mujeres (en kg) } & 58,6 \pm 7,5 \\ \text { Estudiantes de carreras relacionadas con alimentos, nutrición y salud* } & 303(67 \%) \\ \text { Estudiantes de carreras relacionadas a las ciencias sociales y exactas** } & 146(33 \%) \\ & \\ \text { * Carreras: Enfermería, Ingeniería en Alimentos, Ingeniería en Ciencias Agronómicas, Medicina, Nutrición y } & \\ \quad \text { Dietética, Química y Farmacia. } & \end{array}$


Para la elaboración del cuestionario sobre las etapas del cambio referidas al consumo de frutas y verduras, se utilizó el diseñado y validado por el Fondo Internacional para la Investigación del Cáncer (10), actualmente utilizado en Chile en el Programa 5 al Día, previa adaptación y validación en el estudio realizado por Zacarías y cols. (22). Para las preguntas sobre las etapas del cambio referidas a actividad física y control del peso, se aplicaron los instrumentos elaborados y validados por el Programa de Salud Cardiovascular del Ministerio de Salud (23), a partir de los propuestos por Rossi y cols. (24).

Para la elaboración de las preguntas del cuestionario con las que se determinaron las percepciones sobre los beneficios y barreras en actividad física y consumo de frutas y verduras, se utilizaron las propuestas de Eikenberry y cols, (25); Borra y cols. (26); O'Dea y cols. (27), y los resultados del anális is de contenido de los grupos focales con los que se validaron los mensajes sobre consumo de frutas y verduras y actividad física de la Guía para una Vida Saludable 2005 (9), y los resultados del análisis de contenido sobre las motivaciones y barreras con respecto a la actividad física y la alimentación saludable en 8 grupos focales de madres de niños obesos (28).

El instrumento resultante incluyó siete opciones sobre beneficios y barreras en actividad física y consumo diario de frutas y verduras, respectivamente. La encuesta fue validada con 84 estudiantes de Ingeniería en Alimentos, Ingeniería en Ciencias Agronómicas y Química y Farmacia, quienes respondieron en forma anónima y voluntaria. Se evaluó la comprensión de las preguntas, la adecuación de las opciones ofrecidas y el tiempo de aplicación. La totalidad de los estudiantes opinó que las preguntas eran claras y fáciles de contestar. El tiempo de aplicación promedio fue de 7 minutos.

Los estudiantes tuvieron la posibilidad de marcar las opciones correspondientes a sus percepciones y de agregar otras que, según su opinión, representaran mejor sus motivaciones y barreras para lograr cambios de conducta respecto a los dos temas citados. Para evaluar la consistencia interna de las dimensiones del cuestionario se obtuvo un valor del coeficiente alpha de Cronbach de 0.72 . El valor alpha de Cronbach para las motivaciones y barreras en consumo 
de frutas y verduras fue de 0.71 y el valor para las motivaciones y barreras en actividad física y control del peso fue de 0.78 .

Con las opciones originales (todas obtuvieron un porcentaje de respuestas superior al 3\%), más las agregadas por los estudiantes de la prueba piloto (con más de 3\% de respuesta), se construyó el listado de opciones referidas a beneficios y barreras en actividad física y consumo de frutas y verduras, respectivamente. En actividad física, el instrumento incluyó 11 opciones de respuesta para la pregunta "Para mí, las principales razones por las que me resultaría fácil realizar al menos 30 minutos de actividad física 5 veces por semana son (por favor marque todas las que se apliquen a usted)" y 11 opciones de respuesta para la pregunta "Para mí, las principales razones por las que me resultaría difícil realizar al menos 30 minutos de actividad física 5 veces por semana son (por favor marque todas las que se apliquen a usted)".

Respecto al consumo de frutas y verduras, el instrumento incluyó 9 opciones de respuesta para la pregunta "Para mí, las principales razones por las que me resultaría fácil comer 5 porciones de frutas y verduras todos los días son (por favor marque todas las que se apliquen a usted)" y 11 opciones para la pregunta "Para mí, las principales razones por las que me resultaría difícil comer 5 porciones de frutas y verduras todos bs días son (por favor, marque todas las que se apliquen a usted)".

La encuesta fue respondida en forma voluntaria y anónima por los estudiantes, a quienes se solicitó que colocaran su fecha de nacimiento, sexo y carrera que cursaban La estatura y el peso fueron auto reportados por los estudiantes.

\section{Análisis estadístico}

Se determinaron las estadísticas descriptivas de las variables analizadas mediante promedios \pm desviación estándar (DE) en el caso de las variables continuas y se construyeron tablas de frecuencia para las variables categóricas. Para analizar la fiabilidad del cuestionario se realizó una evaluación de la consistencia interna mediante el coeficiente alpha de Cronbach, para el total del cuestionario y para las variables que componen las etapas del cambio. Para evaluar la asociación entre el estado nutricional, etapas del cambio en actividad física, consumo de frutas y 
verduras, y motivaciones y barreras en ambos temas, según género o área de la carrera, se utilizaron la prueba de chi-cuadrado o la prueba exacta de Fisher.

Todos los análisis estadísticos se realizaron utilizando el programa STATA, versión 10 (29) en la Unidad de Estadística y Computación del INTA.

\section{RESULTADOS}

\section{Actividad física}

La figura 1 muestra las etapas del cambio en actividad física en los 445 estudiantes universitarios que contestaron esta pregunta. Se observa que los hombres resultaron significativamente más activos que las mujeres $(\mathrm{p}<0.001)$. En efecto, en la etapa de precontemplación, donde se ubican los que no tienen la intención de realizar actividad física, se encontró a un 5,4\% de los hombres y un 12,4\% de las mujeres; en la etapa de contemplación, que significa tener la intención de realizar actividad física, pero comenzando en unos 6 meses más, se encontró al 15\% de los hombres y al 26\% de las mujeres; y en la etapa de preparación para la acción, donde se encuentran los que tienen la intención de comenzar a realizar actividad física aproximadamente dentro de un mes, se encontró al 23\% de los hombres y al $35 \%$ de hs mujeres. 
Figura 1. Etapas del cambio en actividad física en estudiantes universitarios de Santiago, según género. $\mathrm{N}=445$

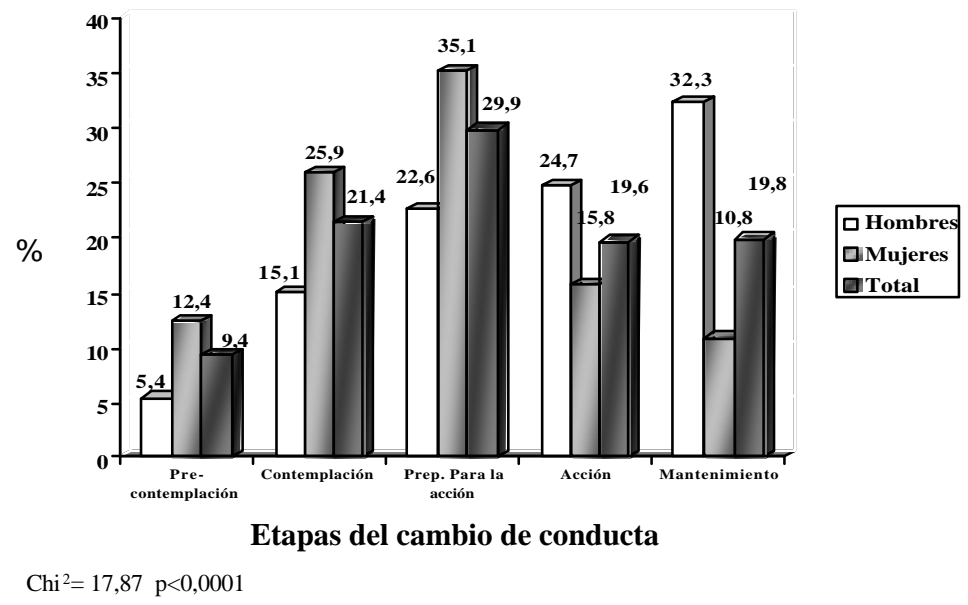

El $25 \%$ de los hombres y el $16 \%$ de las mujeres manifestaron estar realizando actividad física al menos 30 minutos 5 veces o más por semana desde hacía menos de seis meses etapa de acción), en tanto un $32 \%$ de los hombres y un $11 \%$ de las mujeres señaló encontrarse en la etapa de mantenimiento, es decir, realizando actividad física de acuerdo a las condiciones citadas desde hacía más de 6 meses.

$\mathrm{Al}$ analizar su percepción de los beneficios que les reportaba realizar actividad física (tabla 2), se observa que bs principales y en los que los hombres presentaron un significativo mayor porcentaje de respuestas que las mujeres fueron: "me hace sentir bien" $(p<0,01)$, "me entretiene" y "me gusta" ( $\mathrm{p}<0,001)$, en tanto un mayor porcentaje de mujeres manifestó que una de sus motivaciones era "para perder peso" $(p<0,04)$. 
TABLA 2

Beneficios percibidos por estudiantes universitarios de Santiago para realizar al menos 30 minutos de actividad física 5 veces por semana, según género $(\mathrm{N}=449)$.

\begin{tabular}{lcccc|cc}
\multicolumn{1}{c}{ Motivaciones } & \multicolumn{2}{c}{ Hombres } & \multicolumn{2}{c}{ Mujeres } & \multicolumn{2}{c}{ Total } \\
& $\mathbf{N}$ & $\mathbf{\%}$ & $\mathbf{N}$ & $\mathbf{\%}$ & $\mathbf{N}$ & $\%$ \\
Me hace sentir bien & 115 & 61,2 & 129 & 49,4 & 244 & $54,3^{* *}$ \\
Para estar en forma & 101 & 53,7 & 141 & 54,0 & 242 & 53,0 \\
Porque me entretiene & 101 & 53,7 & 96 & 36,8 & 197 & $43,9 * * *$ \\
Porque me gusta & 102 & 54,3 & 90 & 34,5 & 192 & $42,8^{* * *}$ \\
Me hace liberar tensiones & 83 & 44,2 & 101 & 38,7 & 184 & 41,0 \\
Para perder peso & 57 & 30,3 & 103 & 39,5 & 160 & $35,6^{*}$ \\
Para mantener mi peso actual & 39 & 20,7 & 53 & 20,3 & 92 & 20,5 \\
Me permite compartir con amigos & 51 & 27,1 & 30 & 11,5 & 81 & $18,0^{* * *}$ \\
Prueba exacta de Fisher $* \mathrm{p}<0,04 ; * * \mathrm{p}<0,01 ; * * * \mathrm{p}<0,001$ & & & &
\end{tabular}

Las principales barreras percibidas por los estudiantes para realizar al menos 30 minutos de actividad física 5 veces por semana se resumen en la tabla 3. "No tengo tiempo" fue la que obtuvo más respuesta en ambos géneros, en tanto "llego muy cansado/a de la universidad" ( $<<0,004)$, "por flojera" ( $p<0,02)$, "no estoy acostumbrado/a" ( $<<0,001)$, o "no tengo quien me acompañe" $(p<0,004)$, todas con un alto porcentaje de respuesta, fueron significativamente más frecuentes en las mujeres. El 12\% contestó que prefería la TV o el computador, un $10 \%$ indicó que no había seguridad en la calle y un $8 \%$ que no le gustaba, sin diferencias significativas según género. 
TABLA 3

Barreras percibidas por estudiantes universitarios de Santiago para realizar al menos 30 minutos de actividad física 5 veces por semana, según género $(\mathrm{N}=449)$.

\begin{tabular}{|c|c|c|c|c|c|c|}
\hline \multirow[t]{2}{*}{ Barreras } & \multicolumn{2}{|c|}{ Hombres } & \multicolumn{2}{|c|}{ Mujeres } & \multicolumn{2}{|c|}{ Total } \\
\hline & $\mathbf{N}$ & $\%$ & $\mathbf{N}$ & $\%$ & $\mathbf{N}$ & $\%$ \\
\hline No tengo tiempo & 122 & 64,9 & 183 & 70,1 & 305 & 67,9 \\
\hline Llego muy cansado/a de la universidad & 51 & 27,1 & 106 & 40,6 & 157 & $35,0 * *$ \\
\hline Por flojera & 48 & 25,5 & 94 & 36,0 & 142 & $31,6^{*}$ \\
\hline No estoy acostumbrado/a & 37 & 19,7 & 95 & 36,3 & 132 & $29,4 * * *$ \\
\hline Sale muy caro ir a un gimnasio & 35 & 18,6 & 67 & 25,7 & 102 & 22,7 \\
\hline No tengo quien me acompañe & 18 & 9,6 & 52 & 19,9 & 70 & $15,6^{* *}$ \\
\hline Prefiero la TV o el computador & 24 & 12,8 & 28 & 10,7 & 52 & 11,6 \\
\hline No hay seguridad en la calle & 15 & 8,0 & 30 & 11,5 & 45 & 10,0 \\
\hline No me gusta hacer actividad física & 11 & 5,9 & 28 & 10,7 & 39 & 8,7 \\
\hline Tengo problemas de salud & 5 & 2,7 & 9 & 3,5 & 14 & 3,1 \\
\hline
\end{tabular}

\section{Consumo de frutas y verduras}

En lo que respecta al consumo de frutas y verduras, la figura 2 muestra que sólo el $5 \%$ de los estudiantes alcanzaba la meta de 5 porciones diarias, en tanto el $58,4 \%$, consumía 1 a 2 porciones diarias, y el 30,3\% consumía 3 a 4 porciones diarias. Por este motivo, las etapas del cambio tuvieron que ser determinadas de acuerdo al nivel de consumo del grupo mayoritario (12 porciones). 
Figura 2. Número de porciones de frutas y verduras que comen diariamente estudiantes universitarios de Santiago, según género. $\mathrm{N}=449$.

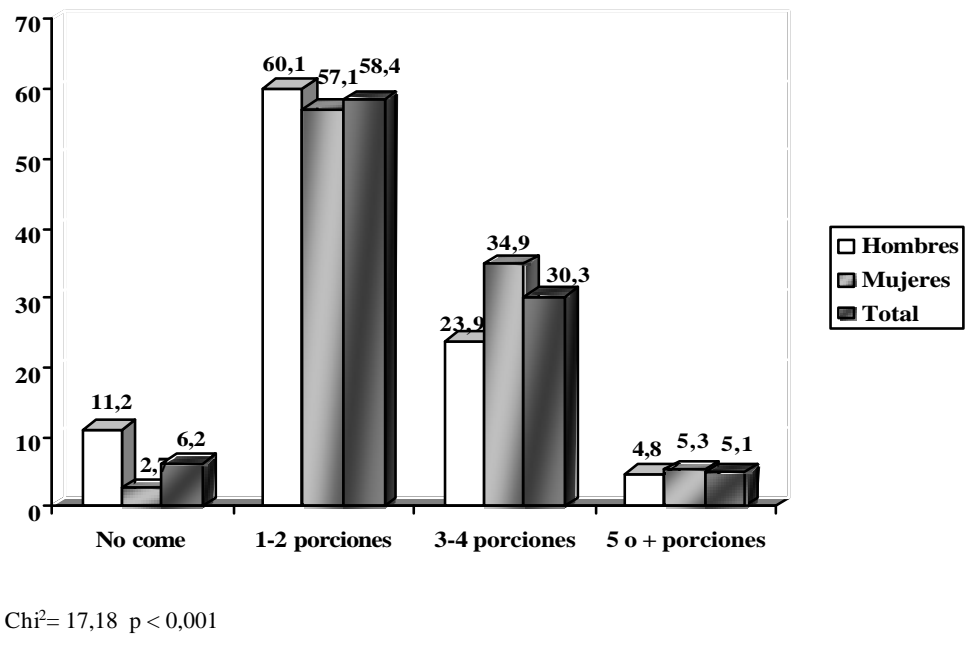

En el caso del 5\% que ya había alcanzado la conducta esperada y consumía 5 porciones al día, la totalidad se encontraba en las etapas de mantenimiento y acción. En el grupo que consumía 1 a 2 porciones, en cambio, el 30\% de hombres y mujeres se encontraba en la etapa de mantenimiento, es decir, estaba consumiendo esa cantidad desde hacía más de 6 meses; el 18\% se encontraba en la etapa de acción, es decir, consumía 1 a 2 porciones diarias desde hacía menos de 6 meses; el 5\% se encontraba en la etapa de preparación para la acción (intención de aumentar su consumo a 5 porciones diarias dentro de un mes); el 9\% se encontraba en la etapa de contemplación, (intención de consumir 5 porciones diarias, pero comenzando en 6 meses más) y el 38\% se encontraba en la etapa de pre-contemplación, es decir, no tenía la intención de aumentar su consumo actual(de 1 a 2 porciones) a 5 porciones diarias. Resultados semejantes se observaron en los estudiantes que consumían 3 a 4 porciones al día.

En cuanto a la percepción de los beneficios de comer 5 porciones diarias de frutas y verduras, los principales fueron: "para estar sano/a y prevenir enfermedades". Cabe hacer notar que esta opción, significativamente más alta en las mujeres $(\mathrm{p}<0,005)$, también tuvo una mayor proporción de respuestas $(\mathrm{p}<0,001)$ en los estudiantes provenientes de las carreras relacionadas a la salud, alimentos y nutrición (datos no mostrados). Las respuestas "me hacen sentir bien" 
$(\mathrm{p}<0,001)$, "me gustan" $(\mathrm{p}<0,005)$ y "para bajar de peso" $(\mathrm{p}<0,001)$, también resultaron significativamente más altas en las mujeres (tabla 4).

\section{TABLA 4}

Beneficios percibidos por estudiantes universitarios de Santiago para comer 5 porciones diarias de verduras y frutas, según género $(\mathrm{N}=449)$.

\begin{tabular}{|c|c|c|c|c|c|c|}
\hline \multirow[t]{2}{*}{ Motivaciones } & \multicolumn{2}{|c|}{ Hombres } & \multicolumn{2}{|c|}{ Mujeres } & \multicolumn{2}{|c|}{ Total } \\
\hline & $\mathbf{N}$ & $\%$ & $\mathbf{N}$ & $\%$ & $\mathbf{N}$ & $\%$ \\
\hline Para estar sano y prevenir enfermedades & 98 & 52,1 & 173 & 66,3 & 271 & $\begin{array}{c}60,4 \\
*\end{array}$ \\
\hline Para sentirme bien & 73 & 38,8 & 147 & 56,3 & 220 & $\begin{array}{c}49,0 \\
* *\end{array}$ \\
\hline Porque me gustan & 76 & 40,4 & 139 & 53,3 & 215 & $\begin{array}{c}47,9 \\
*\end{array}$ \\
\hline Para bajar de peso & 40 & 21,6 & 95 & 36,4 & 135 & $\begin{array}{c}30,1 \\
* *\end{array}$ \\
\hline Para estar en forma & 51 & 27,1 & 79 & 30,3 & 130 & 29,0 \\
\hline Para mantener mi peso actual & 37 & 19,7 & 68 & 26,1 & 105 & 24,0 \\
\hline
\end{tabular}

En la tabla 5, que presenta las barreras planteadas por los y las estudiantes para comer 5 porciones diarias de frutas y verduras, las principales fueron: "vivo solo/a y me da flojera prepararlas", "no tengo tiempo" y "se me olvida comerlas", sin diferencias significativas según género. Las opciones "no me quitan el hambre" $(\mathrm{p}<0,01)$ y "prefiero la comida "chatarra" $(\mathrm{p}<0,001)$, tuvieron un porcentaje de respuesta significativamente mayor en los hombres, en tanto "son difíciles de llevar a la universidad" $(\mathrm{p}<0,01)$ fue respondida por una mayor proporción de mujeres. El 16,5\% de los estudiantes de ambos sexos señaló como barrera "son caras" y el 7\% señaló que no le gustaban las frutas y verduras.

Aunque se encontró que el 77,3\% de los estudiantes tenía un índice de masa corporal menor a 25 (peso normal), un 52,1\% de los hombres y un 78,9\% de las mujeres estaban tratando de bajar o no subir de peso, es decir, se encontraban en las etapas de acción o mantenimiento en lo que respecta al control de su peso $(\mathrm{p}<0,001)$.

No se encontró asociación entre el estado nutricional y las etapas del cambio en actividad física o el consumo de frutas y verduras. 


\section{TABLA 5}

Barreras de estudiantes universitarios de Santiago para comer 5 porciones diarias de verduras $\mathbf{y}$ frutas, según género $(\mathrm{N}=449)$.

\begin{tabular}{lcccccc}
\multicolumn{1}{c}{ Barreras } & \multicolumn{2}{c}{ Hombres } & \multicolumn{2}{c}{ Mujeres } & \multicolumn{2}{c}{ Total } \\
& $\mathbf{N}$ & $\mathbf{\%}$ & $\mathbf{N}$ & $\mathbf{N}$ & $\mathbf{\%}$ \\
Vivo solo/a y me da flojera prepararlas & 66 & 35,1 & 88 & 33,7 & 154 & 34,3 \\
No tengo tiempo & 67 & 35,6 & 83 & 31,8 & 150 & 33,4 \\
Se me olvida comerlas & 58 & 30,9 & 67 & 25,7 & 125 & 27,8 \\
No me quitan el hambre & 49 & 26,1 & 44 & 16,9 & 93 & $20,7^{*}$ \\
Son difíciles de llevar a la universidad & 28 & 14,9 & 64 & 24,5 & 92 & $20,5^{*}$ \\
Son caras & 34 & 18,1 & 40 & 15,3 & 74 & 16,5 \\
Prefiero la "chatarra" & 38 & 20,2 & 22 & 8,4 & 60 & $13,4^{* *}$ \\
No me gustan & 14 & 7,5 & 19 & 7,3 & 33 & 7,4
\end{tabular}

Prueba exacta de Fisher *p<0,01 ** $p<0,001$

\section{DISCUSIÓN}

El estudio de las etapas del cambio (17) en el que se encuentran las personas en relación a una conducta determinada, es ampliamente utilizado para la segmentación de audiencias en las campañas de marketing social que realizan los países desarrollados (18-21). Esto debería ser utilizado también en las intervenciones de promoción de la salud y de educación en nutrición que se desarrollan en el país. El marketing social aplica los principios y técnicas del marketing comercial al análisis, planificación, ejecución y evaluación de programas diseñados para lograr un cambio voluntario de conducta en las audiencias objetivo, con el propósito de mejorar su bienestar personal y el de la sociedad (18).

A diferencia del marketing comercial, cuyo objetivo es posicionar un producto por sobre los de la competencia, en el marketing social los productos se refieren a la conducta saludable que a los planificadores de la campaña les gustaría que la población objetivo o los "consumidores" adoptaran p.e. "realizar 30 minutos diarios de actividad física moderada a intensa" o "consumir al menos 5 porciones de frutas y verduras cada día”. 
Las estrategias educativas o de marketing social que se implementen, para ser exitosas, deben adaptarse a la etapa del cambio de conducta en la que se encuentren las personas a intervenir.

En la ENS 2003 (1), el 23\% de los hombres y el 12\% de las mujeres de 17 a 24 años se declaró activo/a, usando como criterio el que realizaran 30 minutos o más de actividad física fuera del horario de trabajo al menos 3 veces a la semana. En los estudiantes universitarios, en cambio, el $57 \%$ de los hombres y el $22,6 \%$ de las mujeres manifestó estar realizando al menos 30 minutos diario s de actividad física moderada a intensa 5 veces a la semana, criterio actualmente recomendado por el Colegio Americano de la Medicina del Deporte y la Asociación Americana del Corazón (8). Esto significa que los estudiantes varones eran bastante más activos, tanto en relación a la población general, como en relación a las mujeres universitarias.

La actividad física fue objeto de una importante campaña nacional de marketing social en los Estados Unidos de Norteamérica entre los años 2002 a 2006 (30). La campaña Verb TM $_{\text {M }}$ estimuló a los niños de 9 a 13 años a ser físicamente más activos, usando estímulos que resultaran atractivos a ese grupo de edad, como la diversión, la oportunidad de compartir con amigos y la posibilidad de adquirir nuevas habilidades. La campaña combinó ideas, oportunidades y recursos que ayudaran a los niños a incorporar más actividad física a su vida diaria, con el apoyo de instituciones públicas, universidades, organismos no gubernamentales y empresas del sector privado.

La OPS/OMS, a través de la campaña ¡A comer sano, a vivir bien y a moverse América!, está promoviendo el aumento de la xtividad física y la alimentación saludable en toda América Latina, y apoyando proyectos específicos en Brasil, Colombia, Chile y México (22, 31).

En lo que respecta al consumo de frutas y verduras de los estudiantes universitarios, los resultados fueron semejantes a los encontrados por Zacarías y cols. (22), en adultos de NSE medio bajo, asistentes a centros de salud y consumidores de supermercados en Santiago, en el año 2007. En ambos grupos, un bajo porcentaje (5 y 7\%, respectivamente) cumplía la recomendación de consumir al menos 5 porciones diarias y la gran mayoría (58\%) consumía 1 a 2 porciones diarias. Cuando se determinaron las etapas del cambio en los estudiantes 
universitarios que consumían 1 a 2 porciones al día, llamó la atención que el 38\%, en ambos sexos, manifestara no tener intención de aumentar su consumo (etapa de pre-contemplación).

La misma situación se ha observado en otros grupos de edad. Olivares y cols., al comparar el consumo de frutas y verduras en escolares mujeres de NSE medio alto y bajo de Santiago, tampoco encontraron diferencias significativas según NSE, aunque sí encontraron diferencias en el consumo de lácteos (mayor en las de NSE medio alto), pan (mayor en las de NSE bajo) y bocadillos o snacks (mayor en las de NSE bajo) (32).

En Estados Unidos, donde se han observado situaciones similares, se implementó una campaña de marketing social para aumentar el consumo de frutas y verduras en niños y adolescentes, que incluyó su distribución gratuita al interior de los establecimientos educacionales, en las cafeterías, kioscos y máquinas expendedoras, logrando una gran aceptación en todos los grupos (33).

Los países escandinavos, con una larga tradición en políticas públicas que han apoyado el desarrollo de intervenciones que han integrado a los organismos gubernamentales, no gubernamentales, la industria de alimentos y en especial, han considerado la participación de la comunidad, lograron aumentar el consumo de frutas y verduras, de alimentos bajos en grasa y reducir el consumo de sal, con importantes efectos en la prevención de los factores de riesgo de enfermedades cardiovasculares. Este modelo ha sido adoptado por otros países desarrollados en los últimos años (34).

Los beneficios y barreras percibidos por los estudiantes para realizar la actividad física recomendada, muestran que hay diferencias según sexo, necesitando mayor estímulo las mujeres, pero también plantean un desafío a los encargados de las políticas y programas existentes. La falta de tiempo fue el factor más destacado, lo que muestra que no es suficiente con realizar acciones de promoción, es preciso analizar los factores ambientales y determinar si las universidades, el entorno que las rodea y las ciudades están ofreciendo la oportunidad de realizar más actividad física, no sólo a los universitarios, sino a toda la población. 
Situación similar se observa en el consumo de 5 porciones de frutas y verduras. A pesar de que este estudio se realizó en un período de altos precios, producto de las heladas invernales que disminuyeron la disponibilidad de estos productos (año 2007), sólo un 16\% de los estudiantes señaló el costo de las frutas y verduras como barrera. El desarrollo de una estrategia de publicidad similar a la utilizada para los bocadillos (snacks) y otros productos que compiten con las frutas y verduras, podría facilitar que no sólo los estudiantes universitarios, sino todos los grupos que se encuentran en las etapas de mantenimiento, acción, preparación para la acción y aún los que están en la etapa de contemplación, puedan ser motivados a lograr la meta de consumir 5 porciones al día.

Se concluye que tanto la actividad física como el consumo de futas y verduras, dependen en gran medida de las oportunidades que se ofrezcan para lograr los cambios de conducta. Ello significa contar con la participación de actores de los sectores público y privado, que apoyen el desarrollo de la infraestructura y las condiciones para que las personas puedan contar con espacios cercanos y seguros para realizar actividad física, así como con campañas masivas de promoción del consumo de frutas y verduras, para lo que es necesario ampliar y mejorar la oferta de platos preparados en la alimentación institucional y desarrollar nuevos productos procesados en base a frutas y verduras que, manteniendo su gran valor nutricional, sean ofrecidos en envases atractivos, en formato individual y a precios competitivos, cumpliendo con los requisitos de inocuidad, para permitir a los consumidores chilenos alcanzar bs beneficios que se logran al comer al menos 5 porciones al día.

\section{RESUMEN}

Como línea base para diseñar intervenciones de promoción y educación, se determinaron las etapas del cambio, beneficios y barreras relacionados con la actividad física y el consumo de frutas y verduras en 449 estudiantes universitarios de ambos géneros de Santiago. Se utilizaron instrumentos validados internacionalmente y adaptados en el país. En actividad física, los hombres resultaron significativamente más activos que las mujeres. Los beneficios más destacados por ellos fueron "me hace sentir bien" o "me entretiene", en tanto el primero y "perder peso" fueron más frecuentes en las mujeres. En ambos géneros, la principal barrera fue 
la falta de tiempo. El 58\% comía 1 a 2 porciones de frutas y verduras al día y sólo el 5\% alcanzaba las 5 porciones. El principal beneficio de comer frutas y verduras se asoció a "estar sano/a", y las principales barreras fueron "me da flojera prepararlas" o "se me olvida comerlas", en ambos géneros. Estos resultados se utilizarán en el diseño de intervenciones en promoción y educación para estudiantes universitarios.

Palabras claves: estudiantes universitarios, etapas del cambio, actitudes, actividad física, consumo de frutas y verduras.

\section{BIBLIOGRAFIA}

1. Ministerio de Salud. III Encuesta Nacional de Salud. Santiago; MINSAL: 2003.

2. Salinas J, Vio F. Política de promoción de salud en Chile. En: Albala C, Olivares S, Salinas J, Vio F eds. Bases, prioridades y desafíos de la promoción de la salud. Santiago: Ministerio de Salud/Vida Chile/Universidad de Chile/INTA, Andros Impresores; 2004.

3. Lange I, Vio F. Guía para universidades saludables y otras instituciones de educación superior Santiago: Vida Chile, OPS/OMS/INTA/PUC; 2006.

4. World Health Organization. Diet, nutrition and the prevention of chronic diseases: Report of a Joint WHO/FAO Expert Consultation. Geneva: WHO; 2003 (WHO Serial Technical Report; 916).

5. Organización Mundial de la Salud (OMS). Estrategia mundial sobre régimen alimentario, actividad física y salud. 57 ${ }^{\mathrm{a}}$ Asamblea Mundial de la Salud. WHA57.17. Ginebra: OMS; 2004.

6. World Cancer Research Fund. Recommendations for cancer prevention. United Kingdom: WCRF UK; 2007.

7. World Health Organization. A guide for population-based approaches to increasing levels of physical activity. Implementation of the WHO global strategy on diet, physical activity and health. Geneva: WHO; 2007.

8. Haskell WL, Lee IM, Pate RP, Powell KE, Blair SN, Franklin BA, Macera CA, Heath GB, Thompson PD, Bauman A. Physical activity and public kealth. Updated recommendations for 
adults from the American College of Sports Medicine and the American Heart Association. Med Sci Sports Exer 2007; 39: 1423-1434.

9. Ministerio de Salud/INTA/Vida Chile. Guía para una vida saludable. Santiago; Ministerio de Salud; 2005. Norma 76. Disponible en URL: http://www.inta.cl/materiales educativos

10. World Cancer Research Fund/American Institute for Cancer Research. Food, nutrition and the prevention of cancer: a global perspective. Washington DC: WCRF/AICR; 1997.

11. National Institutes of Health/National Cancer Institute. 5 a Day for Better Health Program. Washington DC; Government Printing Office-Monograph: 2004.

12. Ministerio de Agricultura. Oficina de Estudios y Políticas Agrarias (ODEPA). Exportación de frutas y hortalizas. Santiago: ODEPA; 2007. Disponible en URL: http://www.odepa.gob.cl

13. Albala C, Vio F, Kain J, Uauy R. Nutrition transition in Chile: determinants and consequences. Public Health Nutr 2002; 5(1A):123-128.

14. WHO. Marketing of food and non-alcoholic beverages to children. Report of a WHO Forum and Technical Meeting. Oslo, Norway: WHO; 2006.

15. Institute of Medicine of the National Academies. Food marketing to children and youth. Threat or opportunity? McGinnis M, Appleton J, Kraak V, editors. Washington DC: National Academy of Sciences; 2006.

16. Vio F, Zacarías I, González D. Implementación de un programa de promoción del consumo de frutas y verduras. Corporación 5 al Día Chile. En: S Olivares, M Leporati, L Barría, eds. Contribución de la política agraria al consumo de frutas y verduras en Chile: un compromiso con la nutrición y la salud de la población. Santiago: Ministerio de Agricultura/INTA/Corporación 5 al Día Chile; 2008 (páginas 25-42).

17. Prochaska J, Velicer W, Rossi J, Goldstein M, Marcus B et al. Stages of change and decisional balance for twelve problem behaviours. Health Psychology 1994; 13:39-46.

18. Andreasen A. Marketing social change: changing behavior to promote health, social development, and the environment. San Francisco; Jossey-Bass: 1995. 
19. Alcalay R, Bell R. Promoting nutrition and physical activity through social marketing: current practices and recommendations. Center for Advanced Studies in Nutrition and Social Marketing. University of California, Davis. California; 2000.

20. Kotler P, Roberto N, Lee N. Social marketing. Improving the quality of life. $2^{\text {nd }}$ ed. USA: Sage Publications; 2002.

21. Hastings G. Social marketing. Why should the devil have all the best tunes? London: Elsevier Ltd.; 2007.

22. Zacarías I, Lera L, Rodríguez L, Hill R, Domper A, González D. Impact evaluation of a "5 a Day" educational campaign to increase the consumption of fruit and vegetables. Informe Final del Proyecto presentado a OPS/OMS/ILSI/CDC. Santiago: INTA/MINSAL; 2007.

23. Escobar MC, Olivares S, Zacarías I. Manejo alimentario del adulto con sobrepeso y obesidad. Santiago: Ministerio de Salud; 2002.

24. Rossi J, Rossi S, Velicer W, Prochaska J. To change or not to change: That is the question. In: DB Allison. Handbook of the assessment methods for eating behaviors and weight related problems. Newbury Park, CA: Sage, 1995. pp 387-430.

25. Eikenberry N, Smith Ch. Healthful eating: Perceptions, motivations, barriers, and promoters in low-income Minnesota communities. J Am Diet Assoc 2004; 104: 1158-1161.

26. Borra S, Kelly L, Shirreffs M, Neville K, Geiger C. Developing health messages: Qualitatives studies with children, parents, and teachers help identifiy communications oportunities for healthful lifestyles and the prevention of obesity. J Am Diet Assoc 2003;103:721-728.

27. O'Dea J. Why do kids eat healthy food? Perceived benefit of and barriers to healthful eating and physical activity among children and adolescents. J Am Diet Assoc 2003; 103: 497-501.

28. Olivares S, Bustos N, Moreno X, Lera L, Cortez S. Actitudes y prácticas sobre alimentación y actividad física en niños obesos y sus madres en Santiago, Chile. Rev Chil Nutr 2006; 33(2): 170-179.

29. STATA. Stata 10. Stata Corporation. College Station, USA; 2007. 
30. Center for Disease Control and Prevention. National Center for Chronic Disease Prevention and Health Promotion. Atlanta: CDC; 2007. Disponible en URL http://www.cdc.gov.youthcampaign/

31. PAHO/WHO. Re gional communication plan 2006-2008. Let's eat healthy, live well, and get moving America! Washington DC: PAHO; 2006.

32. Olivares S, Bustos N, Lera L, Zelada ME. Estado nutricional, consumo de alimentos y actividad física en escolares mujeres de diferente nivel socioeconómico de Santiago de Chile. Rev Méd Chile 2007; 135: 71-78.

33. Buzby J, Guthrie J, Kantor L. Evaluation of the USDA Fruit and Vegetable Pilot Program: report to Congress. Washington DC: Food Assistance and Nutrition Research Program, Food and Rural Economic Division, Economic Research Service, U.S. Department of Agriculture; 2003.

34. Roos G, Lean M, Anderson A. Dietary interventions in Finland, Norway and Sweden: nutrition policies and strategies. J Hum Nutr Dietet 2002; 15: 19-110. 